\title{
Avaliação das vertentes de abastecimento de água e esgotamento sanitário dos municípios da bacia metropolitana de fortaleza por meio do Índice de Salubridade Ambiental (ISA)
}

\section{Evaluation of water supply and water drainage areas of the metropolitan basin of Fortaleza city through the Environmental Health Index (ISA) \author{
$15 / 08 / 2019$ \\ 29/11/2019
} \\ Data de entrada: \\ Data de aprovação:}

DOI: https://doi.org/10.36659/dae.2021.007

lago Magalhães Praxedes ${ }^{1 *}$ | Antônio Edson Oliveira Marques ${ }^{1}$ | Priscila Alencar Medeiros ${ }^{1}$

Marques AEO (D) https://orcid.org/0000-0001-7177-1564

ORCID ID

Medeiros PA (iD https://orcid.org/0000-0003-2906-1096

\section{Resumo}

O presente trabalho buscou avaliar as vertentes de abastecimento de água e esgotamento sanitário das zonas urbanas dos municípios da Bacia Metropolitana de Fortaleza por meio da aplicação do Índice de Salubridade Ambiental (ISA). Dos 32 municípios que compõem a Bacia Metropolitana, apenas 11 possuem PMSB parcial ou completo. Com relação aos resultados encontrados, dos 11 municípios estudados, apenas o município de Fortaleza encontra-se em situação de salubridade. Para o restante, 54\% foram classificados como Insalubres, $18 \%$ como Baixa Salubridade e $18 \%$ considerados como Média Salubridade. Vale ressaltar que, para o indicador de abastecimento de água, os municípios que apresentaram os melhores resultados foram Fortaleza, Caucaia e Barreira. Já para o indicador de esgotamento sanitário, apenas Fortaleza apresentou resultado satisfatório.

Palavras-chave: Salubridade ambiental. PMSB. ISA.

\section{Abstract}

The present work aimed to evaluate the water supply and sanitation aspects of the urban areas of the municipalities of the Fortaleza Metropolitan Basin by applying the Environmental Health Index (ISA). Of the 32 municipalities that make up the Metropolitan Basin, only 11 have partial or complete PMSB. Regarding the results found, of the 11 municipalities studied, only the municipality of Fortaleza is in a healthy condition. For the remainder, 54\% were classified as Unhealthy, $18 \%$ as Low Salubrity and $18 \%$ considered as Average Salubrity. It is noteworthy that, for the water supply indicator, the municipalities that presented the best results were Fortaleza, Caucaia and Barreira. For the indicator of sanitary sewage, only Fortaleza presented a satisfactory result.

Keywords: Environmental Health. PMSB. ISA.

\footnotetext{
${ }^{1}$ Instituto Federal de Educação, Ciência e Tecnologia do Ceará (IFCE) - Maracanaú - Ceará - Brasil.

* Autor correspondente: iagopraxedes92agmail.com.
} 


\section{INTRODUÇÃO}

Com o advento da Política Nacional de Saneamento Básico (PNSB) instituída pela Lei Federal $n^{\circ} 11.445$, de 5 de janeiro de 2007 (BRASIL, 2007), o setor de saneamento básico ganhou grande destaque entre os gestores municipais brasileiros. As primeiras grandes intervenções nesse setor ocorreram logo após a criação do Plano Nacional de Saneamento (PLANASA), na década de 70, cujo objetivo principal era ampliar a oferta dos serviços de abastecimento de água e esgotamento sanitário por meio de medidas estruturais (obras de intervenção física para conformação da infraestrutura).

A criação da PNSB, marco regulatório do saneamento no Brasil, trouxe um olhar voltado à necessidade da simultaneidade de medidas estruturais com medidas estruturantes (aquelas que fornecem suporte técnico, político e gerencial para a sustentabilidade da prestação dos serviços, necessitando da ação integrada entre os diversos órgãos envolvidos com saneamento). Essa lei estabeleceu as diretrizes nacionais para o saneamento básico, de modo a alcançar a universalização, a integração dos serviços do setor, a promoção da saúde pública e a proteção do meio ambiente.

O saneamento básico pode ser entendido como um conjunto de serviços, infraestruturas e instalações operacionais que visam proporcionar à população saúde pública adequada e a proteção do meio ambiente, garantindo uma maior salubridade ambiental por meio dos serviços de limpeza urbana e manejo dos resíduos sólidos, drenagem e manejo de águas pluviais, abastecimento de água potável e esgotamento sanitário (MORAES et al., 2001; BRASIL, 2007; COSTA, 2010).

Os serviços de abastecimento de água e esgotamento sanitário, historicamente, são considerados as principais vertentes do saneamento pelos gestores municipais, visto que esses serviços são fundamentais para o desenvolvimento econômico, social e industrial de uma região (PHILIPPI JR, MARTINS, 2005; WARTCHOW, 2009). A boa execução desses serviços é uma forma de prevenir problemas ambientais que venham ocasionar malefícios à população, evitando as possíveis ameaças de contaminantes, resíduos, patógenos ou substâncias tóxicas (BRASIL, 2011).

Um dos desafios dos gestores municipais brasileiros é avaliar a prestação desses serviços de modo a garantir seu funcionamento adequado para a população. No art. $9^{\circ}$, inc. I, da PNSB consta que os municípios brasileiros deverão elaborar o seu Plano Municipal de Saneamento Básico (PMSB), devendo englobar todas as vertentes do saneamento, inclusive o abastecimento de água potável e o esgotamento sanitário, como premissa básica para ter acesso aos recursos financeiros da União (BRASIL, 2007; MENDES e LIMA NETO, 2018).

No estado do Ceará, a Política Estadual de Abastecimento de Água e Esgotamento Sanitário (PE$A A E)$, instituída pela Lei Complementar $\mathrm{N}^{\circ} 162$, de 20 de junho de 2016, em consonância com a Política Nacional de Saneamento Básico, veta o apoio financeiro do governo estadual para o município que não tiver estabelecido o PMSB ou plano específico dentro do prazo estipulado (CEARÁ, 2016).

O PMSB é um instrumento de gestão e planejamento que visa apontar as potencialidades e fragilidades dos serviços de saneamento básico do município, bem como estabelecer metas, programas e ações de curto, médio e logo prazo, visando à universalização dos serviços de saneamento (BRASIL, 2007).

Uma necessidade básica dos PMSBs é a criação de instrumentos de monitoramento das ações que envolvem o setor de saneamento (JUNIOR, 2013). Para tanto, a aplicação do Índice de Salu- 
bridade Ambiental (ISA) pode se tornar uma excelente alternativa para suprir essa necessidade. O ISA Foi criado pela Câmara Técnica de Planejamento do Conselho Estadual de Saneamento do Estado de São Paulo (CONESAN) com o intuito de mensurar o grau de salubridade ambiental dos municípios do Estado (SILVA, 2006; SOUZA, 2010). É constituído de indicadores e subindicadores que abrangem as áreas de abastecimento de água, esgotamento sanitário, resíduos sólidos, recursos hídricos e controle de vetores (SILVA, 2006; COSTA, 2010).

Uma das vantagens da adoção do ISA em detrimento de outros indicadores ambientais está na facilidade de compilar os dados referentes ao saneamento em uma só informação, fazendo com que ele seja uma ferramenta de consolidação dos fatores ambientais prioritários, permitindo uma análise abrangente da prestação dos serviços e facilitando seu acompanhamento, garantindo uma maior uniformidade das informações (COSTA, 2010). Dessa forma, o ISA se mostra como uma ferramenta importante de implantação e monitoramento de políticas públicas, além de servir como medida preventiva à degradação ambiental (BESSA JR e MULLER, 2000; NEUMAN, CALMON e AGUIAR, 2013).

Assim, esse trabalho tem como objetivo avaliar as vertentes de abastecimento de água e esgotamento sanitário nas zonas urbanas da Bacia Metropolitana no Ceará por meio dos PMSB já elaborados, utilizando-se os subindicadores do ISA de cobertura de abastecimento de água, qualidade da água distribuída, saturação do sistema produtor de água, cobertura de coleta de esgotos e tanques sépticos, tratamento dos efluentes e saturação dos sistemas de esgotamento sanitário, mostrando um panorama geral dessas duas vertentes nos municípios dessa bacia.

\section{METOdOLOGIA}

A Bacia Metropolitana detém a maior parte da população do estado do Ceará e possui os maiores aportes de investimentos, uma vez que nessa região hidrográfica estão inseridas a capital Fortaleza e parte dos municípios litorâneos, representando um alto potencial turístico.

Essa bacia fica localizada na porção nordeste do Estado do Ceará, sendo formada por 16 sub-bacias, destacando-se as dos rios Choró, Pacoti, São Gonçalo, Pirangi, Ceará e Cocó, pois possuem as maiores extensões. Essa região hidrográfica possui uma área de $15.085 \mathrm{~km}^{2}$, correspondendo a 10\% do território do estado (CEARÁ, 2009).

A bacia é responsável por drenar totalmente os municípios de Acarape, Aquiraz, Aracoiaba, Barreira, Baturité, Beberibe, Capistrano, Cascavel, Caucaia, Choro, Chorozinho, Eusébio, Fortaleza, Guaiúba, Horizonte, Itapiúna, Itaitinga, Maracanaú, Ocara, Pacajus, Pacatuba, Pindoretama, Redenção; e parcialmente os municípios de Aracati (8,97\%), Aratuba (83,40\%), Canindé $(20,10 \%)$, Fortim (65,61\%), Guaramiranga (82,24\%), Ibaretama (87,07\%), Maranguape (94,03\%), Morada Nova (22,72\%), Mulungu (65,04\%), Pacoti $(95,05 \%)$, Palhano $(40,47 \%)$, Palmácia (94,66\%), Paracuru (17,80\%), Pentecoste (29,03\%), Quixadá (21,82\%), Russas (14,02\%) e São Gonçalo do Amarante (64,46\%). Na Fig. 1 é possível observar a área drenada pela bacia, bem como a sua localização. 


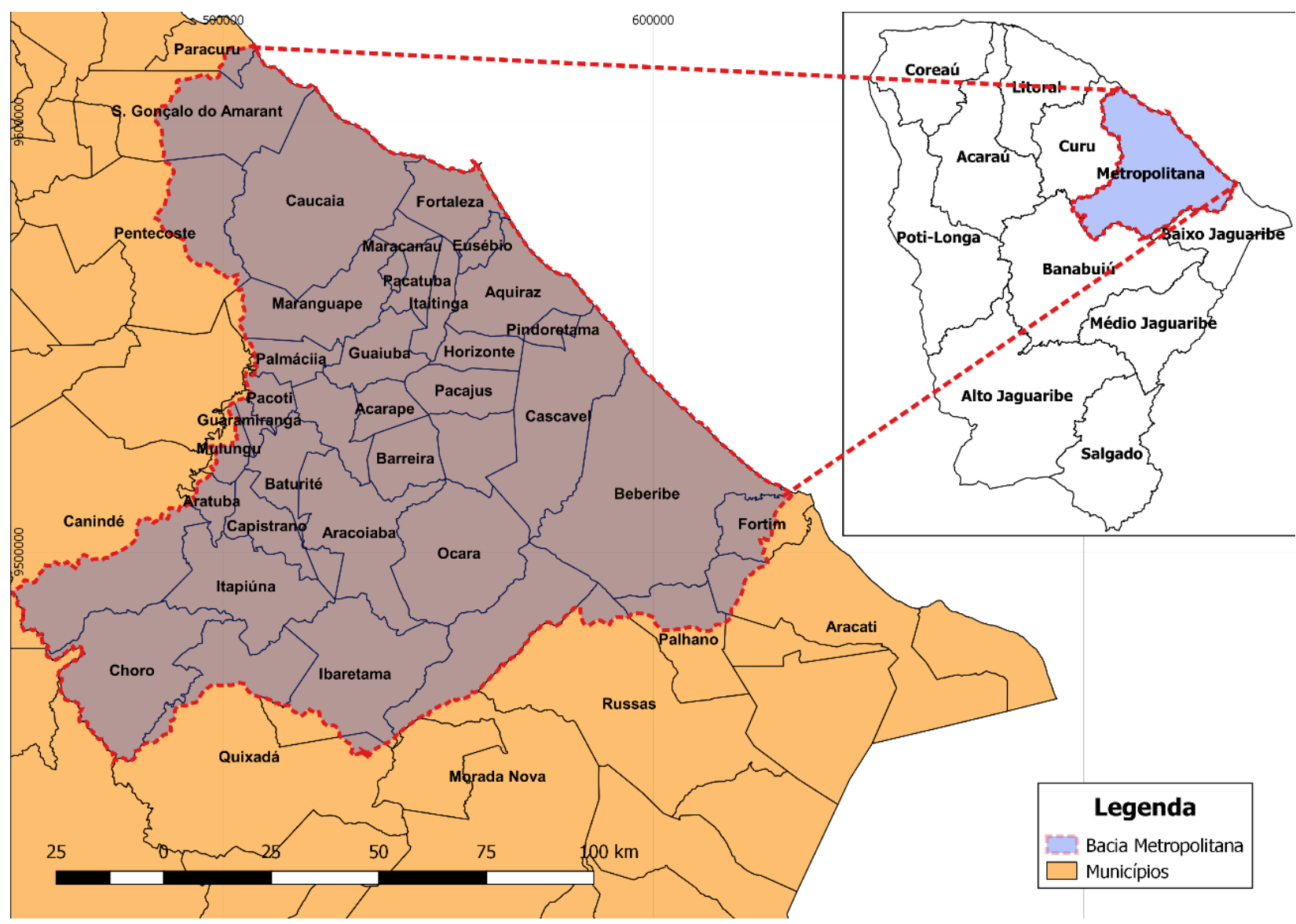

Figura 1 - Mapa de localização da Bacia Metropolitana e municípios drenados Fonte: IBGE, 2019; COGERH, 2019.

Os municípios que irão englobar os resultados dessa pesquisa são aqueles que possuem PMSB completo ou específico para as vertentes abastecimento de água e esgotamento sanitário, visto que, na PNSB e no PEAAE, o acesso aos recursos da União ou do Estado só poderá ser liberado caso os mesmo possuam o plano. Quanto ao prazo para adequação aos instrumentos legais citados, tem-se no Decreto $n^{\circ} 9.254$ de 2017 que os municípios que não elaborarem seu PMSB até dezembro de 2019 não poderão ter acesso aos recursos supracitados.

Os municípios da Bacia Metropolitana que possuem PMSB completo são: Aratuba, Barreira, Be- beribe, Caucaia, Eusébio, Fortaleza, Horizonte e Mulungu; parcial (água e esgoto), Aquiraz, Cascavel e Redenção.

Além das informações contidas nos PMSBs dos municípios citados, foram utilizados os dados do censo do IBGE de 2010 e da Companhia de Água e Esgoto do Ceará (Cagece) ${ }^{1}$ do ano de 2018.

\subsection{ISA - Água e Esgoto}

O ISA aplicado neste trabalho visou abordar as zonas urbanas dos municípios que possuem PMSB, visto que os indicadores utilizados nos

Volumes consumidos de água e coletados de esgoto, perdas nos sistemas de distribuição de água, população abastecida com água e esgoto. 
cálculos são característicos dessas áreas (CONESAN, 1999; COSTA, 2010). O ISA de água e esgoto foi elaborado conforme a Eq. 1:

$\mathrm{ISA}_{(\mathrm{AE})}=0,65 \mathrm{lab}+0,35 \mathrm{les}$

Onde: lab - Indicador de Abastecimento de Água; les - Indicador de Esgotamento Sanitário.

$\mathrm{Na}$ Eq. 1 foi adotado peso maior para o abastecimento de água, uma vez que essa vertente é fundamental para o desenvolvimento econômico, social e industrial de uma região, como proposto no trabalho de Mendes e Lima Neto (2018).

Para cada município analisado, foi calculado o ISA para o abastecimento de água e esgotamento sanitário. Foi verificada inicialmente a divisão administrativa de cada município (a nível de distrito) que consta no Censo do IBGE (BRASIL, 2010), bem como identificados os sistemas que atendem às zonas urbanas dos respectivos distritos e o operador responsável por esses sistemas. No total, foram analisados 47 distritos, 14 sistemas de distribuição de água e 12 sistemas de esgotamento sanitário. Os resultados da análise desagregada substanciaram a obtenção do ISA integral do município.

As pontuações resultantes da aplicação da Eq. 1 permitiram a interpolação e o enquadramento nas faixas apresentadas na Tabela 1. Tal enquadramento possibilita a classificação da condição de salubridade da localidade em estudo e, posteriormente, do município.
Tabela 1 - Faixa de situação de salubridade (\%).

\begin{tabular}{|c|c|}
\hline Condição de Salubridade & Pontuação (\%) \\
\hline Insalubre & $0-25$ \\
\hline Baixa Salubridade & $25-50$ \\
\hline Média Salubridade & $50-75$ \\
\hline Salubre & $75-100$ \\
\hline \multicolumn{2}{|c|}{ Fonte: Adaptado de BATISTA e SILVA, 2006. }
\end{tabular}

Os valores observados na Tabela 1 foram adaptados de Batista e Silva (2006) para melhor representar os resultados encontrados. A adaptação se refere aos valores da faixa de salubridade.

\subsection{Indicador de Abastecimento de Água $\left(I_{a b}\right)$}

$\mathrm{O} \mathrm{I}_{\mathrm{ab}}$ é um indicador que visa avaliar o abastecimento de água de uma região a partir de subindicadores que abrangem: i) os domicílios atendidos com abastecimento de água de forma adequada; ii) a qualidade da água oferecida conforme os valores máximos permitidos pela Portaria de consolidação $\mathrm{N}^{\circ} 5$, de 28 de setembro de 2017, do Ministério da Saúde; iii) a capacidade de produção das Estações de Tratamento de Água. $\mathrm{Na}$ Eq. 2 é possível observar os subindicadores contabilizados para o cálculo do $\mathrm{I}_{\mathrm{ab}}$.

$$
\mathrm{Iab}=\frac{\mathrm{Ica}+\mathrm{Iqa}+\mathrm{Isa}}{3} \times 100
$$

Onde: I - Indicador Cobertura de Abastecimento; $I_{\text {qa }}$ - Indicador de qualidade de água distribuí$\mathrm{da} ; \mathrm{I}_{\mathrm{sa}}$ - Indicador de saturação do sistema produtor (quantidade).

Na Tabela 2 é possível observar a metodologia de cálculo dos subindicadores que compõem o $\mathrm{I}_{\mathrm{ab}}$. 
Tabela 2 - Metodologia de cálculo dos subindicadores do $\mathrm{I}_{\mathrm{ab}}$.

\begin{tabular}{|c|c|c|}
\hline Subindicador & Variáveis & Pontuação \\
\hline $\mathrm{Ica}=\frac{\text { Dua }}{\text { Dut }} \cdot 100$ & $\begin{array}{l}\mathrm{D}_{\text {ua }}=\text { domicílios urbanos atendidos; } \\
\mathrm{D}_{\mathrm{ut}}=\text { domicílios urbanos totais. }\end{array}$ & (\%) direto pela fórmula \\
\hline $\mathrm{Iqa}=\mathrm{K} \cdot \frac{\mathrm{Naa}}{\mathrm{Nar}} \cdot 100$ & $\begin{array}{c}\mathrm{K}=\mathrm{n}^{\circ} \text { amostras realizadas } / \mathrm{n}^{\circ} \text { de amostras exigidas pelo serviço de } \\
\quad \text { abastecimento de água; } \\
\qquad \begin{array}{c}\mathrm{N}_{\mathrm{aa}}=\mathrm{n}^{\circ} \text { amostras consideradas potáveis; } \\
\mathrm{N}_{\mathrm{ar}}=\mathrm{n}^{\circ} \text { amostras realizadas (mensais). }\end{array}\end{array}$ & $\begin{array}{c}I_{\text {qa }}=100 \% \text { Pont. } 100 \\
95 \leq I_{q a} \leq 99 \% \text { Pont. } 80 \\
85 \leq I_{\text {qa }} \leq 94 \text { Pont } 60 \\
70 \leq I_{q a} \leq 84 \text { Pont } 40 \\
50 \leq I_{\text {qa }} \leq 69 \text { Pont } 20 \\
I_{q a} \leq 49 \text { Pont. } 0\end{array}$ \\
\hline $\mathrm{Isa}=\mathrm{n}=\frac{\left(\log \frac{\mathrm{Cp}}{\mathrm{Vp} \cdot \overline{\mathrm{K} 2}}\right)}{\log (1+\mathrm{t})}$ & $\begin{array}{c}\mathrm{n}=\mathrm{n}^{\circ} \text { de anos em que o sistema ficará saturado } \\
\mathrm{V}_{\mathrm{p}}=\text { volume de produção para atender a } 100 \% \text { da população atual } \\
\mathrm{C}_{\mathrm{p}}=\text { capacidade de produção } \\
\mathrm{t}=\text { taxa de crescimento anual média da população } \\
\text { para os próximos } 5 \text { anos } \\
\mathrm{K}_{1}=\text { coeficiente de perda atual } \\
\mathrm{K}_{2}=\text { coeficiente de perda prevista para } 5 \text { anos }\end{array}$ & $\begin{aligned} \mathrm{n} & \geq 5 \text { Pont. } 100 \\
0<\mathrm{n} & <5 \text { Pont. Interpolar } \\
\mathrm{n} & \leq 0 \text { Pont. } 0\end{aligned}$ \\
\hline
\end{tabular}

Fonte: CONESAN, 1999.

$\mathrm{O} \mathrm{I}_{\text {ca }}$ tem por objetivo avaliar a quantidade de domicílios que são atendidos adequadamente pelo abastecimento de água. Neste trabalho foi considerado abastecimento adequado os domicílios atendidos por rede e cisterna, conforme adotado pelo Plano Nacional de Saneamento Básico (Plansab). Não foi considerado o abastecimento de água por poços, uma vez que não se pode garantir o abastecimento contínuo de água nem a garantia da qualidade da água oferecida.

$\mathrm{O}$ indicador $\mathrm{I}_{\text {qа }}$ visa monitorar a qualidade de água fornecida pelos sistemas produtores, avaliando os parâmetros de Turbidez, Cor e Cloro Residual Livre. Esses dados foram disponibilizados pela Cagece e se referem ao ano de 2018. Vale ressaltar que os distritos que não são abastecidos por sistema de abastecimento de água tiveram seus valores de $\mathrm{I}_{\mathrm{qa}}$ zerados, visto que não é possível classificar a segurança sanitária dessa água.

Por fim, o $\mathrm{I}_{\mathrm{sa}}$ tem por objetivo comparar a oferta e demanda de água, visando relatar, se houver, a necessidade de construção de novos Sistemas de Abastecimento de Água (SAA's) para atender às populações futuras. Os dados de projeção da população e perdas nos sistema foram retirados dos PMSB's dos municípios analisados. Para a variável $V_{p}$, os dados foram retirados do Relatório Anual de Dados Operacionais (Radop) fornecidos pela CAGECE. Por sua vez, a variável $C_{p}$ foi calculada com base na metodologia adotada no PMSB de Fortaleza, conforme explicitado na Eq. 3.

$C_{p}=\frac{P \times q}{(1-\text { ip) } \times 86400} \times 100$

Onde: P - População urbana; q - Consumo de água per capita; ip - Índice de perda.

\section{$2.3 \mathrm{I}_{\mathrm{es}}$ - Indicador de Esgotamento Sanitário}

$O \mathrm{I}_{\mathrm{es}}$ é um indicador que visa avaliar a população atendida pelo sistema de esgotamento sanitário de uma região a partir de subindicadores que abrangem: i) os domicílios atendidos com esgotamento sanitário de forma adequada; ii) se o efluente é coletado e tratado; e iii) a capacidade de tratamento dos Sistemas de Esgotamento Sanitário (SES). Na Eq. 4 é possível observar os subindicadores contabilizados para o cálculo do $\mathrm{I}_{\mathrm{es}}$.

Ies $=\frac{\text { Ice }+ \text { Ite }+ \text { Ise }}{3} \times 100$

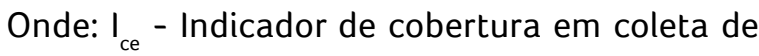
esgotos e tanques sépticos; $I_{\text {te }}$ - Indicador de tratamento de esgotos e tanques sépticos; se $_{\text {se }}$ Indicador de saturação do tratamento. 
Na Tabela 3 é possível observar a metodologia de cálculo dos subindicadores que compõem o les.

Tabela 3 - Metodologia de cálculo dos subindicadores do $\mathrm{I}_{\mathrm{es}}$.

\begin{tabular}{|c|c|c|}
\hline Subindicador & Variáveis & Pontuação \\
\hline Ice $=\frac{\text { Due }}{\text { Dut }} \cdot 100$ & $\begin{array}{l}D_{\mathrm{ue}}=\text { domicílios urbanos atendidos por coleta ou tanque séptico; } \\
\qquad D_{\mathrm{ut}}=\text { domicílios urbanos totais. }\end{array}$ & $\begin{array}{c}I_{c e} \geq 85 \% \text { Pont. } 100 \\
65 \leq I_{\text {ce }}<85 \% \text { Interpolar } \\
\text { I ce }_{\text {ce }}<65 \% \text { Pont. } 0\end{array}$ \\
\hline $\mathrm{Ite}=\mathrm{Ice} \cdot \frac{\mathrm{Vt}_{\mathrm{t}}}{\mathrm{V}_{\mathrm{c}}} \cdot 100$ & $\begin{array}{l}\mathrm{V}_{\mathrm{t}}=\text { volume tratado; } \\
\mathrm{V}_{\mathrm{c}}=\text { volume coletado. }\end{array}$ & $\begin{array}{c}I_{\text {te }} \geq 72,25 \% \text { Pont. } 100 \\
26 \leq I_{\text {te }} \leq 72,24 \% \text { Interpolar } \\
\left.\right|_{\text {te }}<26 \% \text { Pont } 0 \\
70 \leq I_{\text {qa }} \leq 84 \text { Pont } 40\end{array}$ \\
\hline $\mathrm{Ise}_{\mathrm{se}}=\frac{\left(\log \frac{\mathrm{Ct}}{\mathrm{V}_{\mathrm{c}}}\right)}{\log (1+\mathrm{t})}$ & $\begin{array}{c}\mathrm{n}=\mathrm{n}^{\circ} \text { de anos em que o sistema ficará saturado; } \\
V_{\mathrm{c}}=\text { volume coletado de esgoto; } \\
\mathrm{C}_{\mathrm{t}}=\text { capacidade de tratamento; } \\
\mathrm{t}=\text { taxa de crescimento anual média da população } \\
\text { para os próximos } 5 \text { anos. }\end{array}$ & $\begin{array}{c}\mathrm{n} \geq 3 \text { Pont. } 100 \\
0<\mathrm{n}<3 \text { Pont. Interpolar } \\
\mathrm{n} \leq 0 \text { Pont. } 0\end{array}$ \\
\hline
\end{tabular}

Fonte: CONESAN, 1999.

$\mathrm{OI}_{\text {ce }}$ tem por objetivo avaliar a quantidade de domicílios que são atendidos adequadamente pelo sistema de esgotamento sanitário.

$O$ indicador $I_{\text {te }}$ visa quantificar e qualificar os domicílios atendidos por rede de esgoto e fossa séptica. Foi considerado que o $V_{c}$ é $80 \%$ do volume consumido de água. Já para o cálculo do $\mathrm{V}_{\mathrm{t}}$ adotou-se que o volume coletado pelos domicílios atendidos é igual ao volume tratado.

$\mathrm{O} \mathrm{I}_{\mathrm{se}}$ avalia a capacidade de tratamento das Estações de Tratamento de Esgoto e visa relatar, se houver, a necessidade de construção de novos SES's ou construções de fossas sépticas para atender às demandas das populações futuras. Para os distritos que não possuem SES, foi adotado para o cálculo do $C_{t}$ valor de volume médio de $130 \mathrm{~L} / \mathrm{ha}$ bitante de geração de efluentes, conforme Norma da ABNT - Associação Brasileira de Normas Técnicas NBR 7229/2003 - Padrão Médio.

\section{RESULTADOS E DISCUSSÃO}

Os valores encontrados dos subindicadores e do ISA(AE), bem como a faixa de situação de salubridade para os municípios de Aratuba, Aquiraz, Barreira, Beberibe, Caucaia, Cascavel, Eusébio, Fortaleza, Horizonte, Mulungu e Redenção podem ser vistos na Tabela 4.

Tabela 4 - Resultados dos subindicadores e do ISA(AE) dos municípios estudados.

\begin{tabular}{|c|c|c|c|}
\hline Municípios & I $_{\text {ab }}$ & I $_{\text {es }}$ & Situação \\
\hline Fortaleza & 86,20 & 73,69 & 81,82 \\
\hline Caucaia & 82,79 & 0,07 & 53,84 \\
\hline Barreira & 77,36 & 0,00 & 50,28 \\
\hline Eusébio & 51,19 & 0,00 & 33,27 \\
\hline Redenção & 45,57 & 0,00 & 29,62 \\
\hline Horizonte & 38,45 & 0,00 & 24,99 \\
\hline Aratuba & 33,01 & 0,00 & 21,46 \\
\hline Mulungu & 32,76 & 0,00 & 21,30 \\
\hline Cascavel & 31,51 & 0,00 & 20,48 \\
\hline Aquiraz & 25,63 & 10,59 & Baixa \\
\hline Beberibe & 26,48 & 0,00 & Insalubre \\
\hline
\end{tabular}


O Indicador de Abastecimento de Água $\left(\mathrm{I}_{\mathrm{ab}}\right)$ apresentou uma variação bastante significativa durante o período analisado, com pontuações entre 25,63 e 86,20 . Os municípios que apresentaram melhores resultados foram Fortaleza $(86,20)$, Caucaia $(82,79)$ e Barreira $(77,36)$. Esse fator pode ser explicado pelo alto índice cobertura de água desses municípios (mais de 96\%) e pela capacidade de produção dos sistemas integrados. Resultados semelhantes foram relatados nos trabalhos de Valvassori e Alexandre (2012) e Lins e Moraes (2017).

Notou-se neste trabalho que os municípios abastecidos por Sistemas Integrados (SI) apresentaram os melhores resultados para Indicador de saturação do sistema produtor (Isa). Isso mostra que os SI garantem uma boa capacidade de produção de água, uma vez que os mananciais utilizados para esses sistemas possuem maior capacidade de acumulação, o que pode ser notado nos dados fornecidos pela Cagece (CEARÁ, 2018).

Para o restante dos municípios, houve uma tendência dos sistemas de abastecimento de água não estarem fornecendo água em quantidade e qualidade suficientes para o abastecimento humano, apresentando, na maior parte desses, sistemas produtores já saturados e com ineficiência na capacidade de tratamento. Isso serve de alerta para os gestores públicos priorizarem os investimentos para essas áreas e buscar novas alternativas para o abastecimento de água dessas regiões.

Em menção ao Indicador de Esgotamento Sanitário $\left(\mathrm{I}_{\mathrm{es}}\right)$, apenas os municípios de Aquiraz, Caucaia e Fortaleza obtiveram valores maiores do que zero, ressaltando-se Fortaleza, que obteve uma pontuação de 73,69, garantindo uma boa salubridade para a população dessa cidade. Esses resultados foram melhores do que os encontrados por Pinto et al. (2016) para o município de Diamante do Oeste - PR e piores do que os resultados encontrados por Batista e Silva (2006), que avaliaram o município de João Pessoa - PB.

A cobertura de coleta e tratamento de esgoto (considerando rede de esgoto e fossa séptica) variou entre 13,89 e $75,69 \%$, sendo o pior resultado inferido para o município de Cascavel e o melhor resultado para a cidade de Fortaleza. Vale ressaltar que nove dos 11 municípios estudados apresentaram índices de cobertura abaixo dos $50 \%$, demostrando uma situação preocupante quanto aos aspectos sanitários da cidade.

Por fim, diante do exposto na Tabela 4, o valor do ISA $_{(\mathrm{AE})}$ para os municípios avaliados variou entre 17,21 e 81,82. Na Fig. 2 é possível observar como se comportou a faixa de salubridade nessas áreas. 


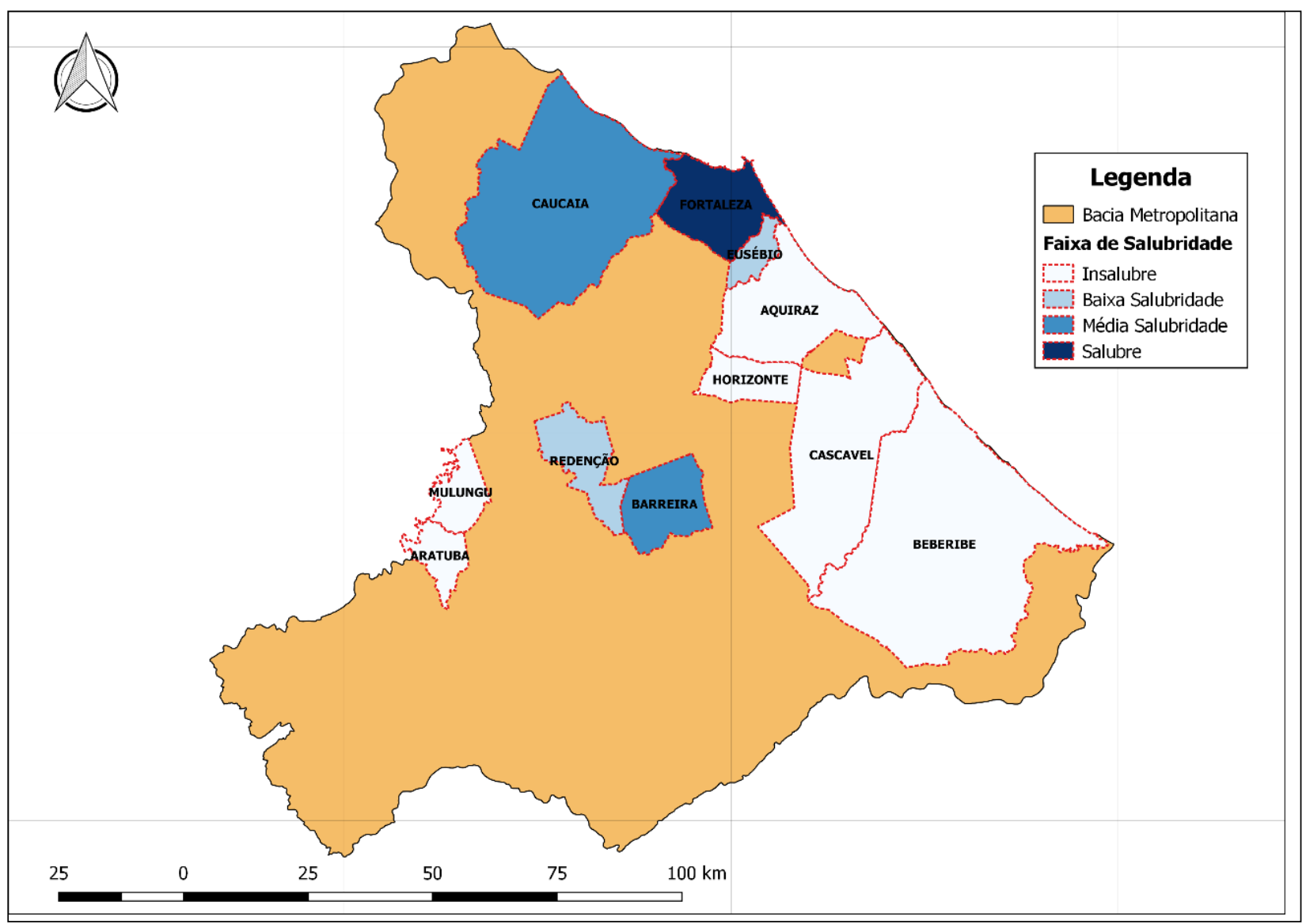

Figura 2 - Faixa de salubridade dos municípios estudados

Conforme a Fig. 2, pode-se constatar que, dos municípios estudados, $54 \%$ foram classificados como Insalubres, $18 \%$ como Baixa Salubridade e $18 \%$ considerados como Média Salubridade. Apenas Fortaleza obteve classificação como Salubre. Esses resultados foram piores do que os relatados por Mendes e Lima (2018), que avaliaram a salubridade ambiental dos municípios das bacias hidrográficas do Alto Jaguaribe e Salgado, no estado do Ceará.

Para os municípios classificados como insalubres ou com baixa salubridade, deve-se haver a priorização de investimentos públicos, pois essas áreas se encontram em situação crítica de salubridade, podendo afetar o meio ambiente e a saúde da população.

\section{CONCLUSÃO}

Os resultados obtidos para o Indicador de Abastecimento de Água (lab) variaram entre 25,63 e 86,20 , com os municípios de Fortaleza, Caucaia e Barreira apresentando as melhores pontuações. Já os municípios que apresentaram os piores resultados foram Beberibe e Aquiraz.

Com relação ao Indicador de Esgotamento Sanitário (les), apenas Fortaleza apresentou boa pontuação. O restante dos municípios apresentou resultado zero ou próximo dele. Isso mostra que os aspectos sanitários dessas áreas estão comprometidos, colocando em risco a saúde da população urbana mediante a disseminação de doenças por veiculação hídrica e degradação do meio ambiente. 
Quanto ao ISA(AE), dos municípios estudados, $54 \%$ foram classificados como Insalubres, $18 \%$ como Baixa Salubridade e 18\% considerados como Média Salubridade, sendo apenas o município de Fortaleza com situação Salubre.

Vale ressaltar que, dos 32 municípios que que compõem a Bacia Metropolitana, apenas 11 possuem PMSB completo ou parcial. Por essa razão, este trabalho não pôde abranger todo o território da bacia hidrográfica.

Por fim, conforme a metodologia adotada, fica claro que deve haver maiores investimentos no tocante ao saneamento básico dessa região, principalmente na vertente de esgotamento sanitário, já que 10 dos 11 municípios avaliados não apresentaram resultados satisfatórios.

\section{CONTRIBUIÇÃO DOS AUTORES}

Todos os autores contribuíram de forma igualitária.

\section{REFERÊNCIAS}

BATISTA, M. E. M.; SILVA, T. C. O Modelo ISA/JP - Indicador de Performance para diagnóstico do saneamento ambiental urbano. Engenharia Sanitária e Ambiental, Rio de Janeiro, v. 11, n. 1, p.55-64, mar. 2006. Disponível em: <http://www.scielo.br/scielo.php?script $=$ sci_arttext $\&$ pid $=$ S1413-41522006000100008>. Acesso em: 03 abr. 2019. https://doi.org/10.1590/S141341522006000100008

BESSA JR., O.; MÜLLER, A. C. P. Indicadores Ambientais Georreferenciados para a Área de Proteção Ambiental de Guaraqueçaba. Revista Paranaense Desenvolvimento. Curitiba, n. 99, p. 105119, jul/dez 2000. Disponível em: <http://www.ipardes.pr.gov. br/ojs/index.php/revistaparanaense/article/view/239> Acesso em: 10 jun. 2019.

BRASIL. Lei n ${ }^{\circ}$ 11.445, de 05 de janeiro de 2007. Estabelece Diretrizes Nacionais para o Saneamento Básico; Altera as Leis nos 6.766, de 19 de Dezembro de 1979, 8.036, de 11 de Maio de 1990, 8.666, de 21 de Junho de 1993, 8.987, de 13 de Fevereiro de 1995; Revoga a Lei no 6.528, de 11 de Maio de 1978; e dá outras providências. Diário Oficial da União, 8 de janeiro de 2007. Brasília, 2007.
BRASIL. Instituto Brasileiro de Geografia e Estatística - IBGE. Atlas do Saneamento 2011. Qualidade e eficiência dos serviços de saneamento. Rio de Janeiro: IBGE, 2011. p. 27-28.

BRASIL. Decreto $n^{\circ} 9.254$, de 29 de dezembro de 2017. Altera o Decreto $\mathrm{n}^{\circ} \mathbf{7 . 2 1 7}$, de 21 de junho de 2010, que regulamenta a Lei $n^{\circ} 11.445$, de 5 de janeiro de 2007, que estabelece diretrizes nacionais para o saneamento básico. Diário Oficial da União, 29 de dezembro de 2017. Brasília, 2017.

BRASIL. Ministério da Saúde. Portaria de Consolidação $n^{\circ} 5$, de 28 de setembro de 2017. Em seu Anexo XX trata-se do controle e da vigilância da qualidade da água para consumo humano e seu padrão de potabilidade. Diário Oficial da União, 03 de outubro de 2017. Brasília, 2017.

Ceará. Assembleia Legislativa. Caderno regional das bacias Metropolitanas. SANTANA, E. W. (Coordenador). Fortaleza, INESP, 2009. 136p. v. 9. Disponível em: <http://docplayer.com. br/24976975-Caderno-regional-das-bacias-metropolitanas. html> Acesso em: 09 jun. 2019.

CEARÁ. Lei Complementar N 162, de 20 de junho de 2016. Institui a Política Estadual de Abastecimento de Água e de Esgotamento Sanitário no Estado do Ceará, institui o Sistema Estadual de Abastecimento de Água e Esgotamento Sanitário, o Sistema Estadual de Informações em Saneamento, e cria o Fundo Estadual de Saneamento. Diário Oficial do Estado, 22 de junho de 2016. Fortaleza, 2016.

COSTA, R. V. F. Desenvolvimento do Índice de Salubridade ambiental (ISA) para comunidades rurais e sua aplicação e análise nas comunidades de Ouro Branco - MG. 2010. 186 f. Dissertação (Mestradoem Engenharia Ambiental), Universidade Federal de Ouro Preto, Ouro Preto, 2010. Disponível em: <https://www.repositorio.ufop.br/bitstream/123456789/2287/1/ DISSERTA\%c3\%87\%c3\%830_Desenvolvimento\%c3\%8dndiceSalubridade.pdf>. Acesso em: 03 jul. 2019.

JUNIOR, A. C. G. Planos Municipais de Saneamento Básico. Revista DAE, São Paulo, v. 1, n. 193, p.6-15, dez. 2013. Disponível em: <http://revistadae.com.br/downloads/edicoes/RevistaDAE-193.pdf>. Acesso em: 03 abr. 2019.

LINS, A. F.; MORAES, A. R. Determinação do Índice de Salubridade Ambiental no município de Guaíra-PR, Brasil. In: Congresso Brasileiro de Gestão Ambiental, 8., 2017, Campo Grande. Anais.... Campo Grande: Ibeas, 2017. p. 1 - 11. Disponível em: <https:// www.ibeas.org.br/congresso/Trabalhos2017/IX-003.pdf>. Acesso em: 05 jun. 2019.

MENDES, J. S.; LIMA NETO, I. E. Análise e projeção da salubridade ambiental com base em planos municipais de saneamento básico. Revista DAE, São Paulo, v. 66, n. 210, p.5-16, jun. 2018. Disponível em: <http://revistadae.com.br/artigos/artigo_edicao_210_n_1717.pdf>. Acesso em: 21 fev. 2019. https://doi. org/10.4322/dae.2018.002 
MORAES, R. S. et al. Plano Municipal de Saneamento Ambiental de Alagoinhas: Metodologia e Elaboração. Santo André, SP: SEMASA Saneamento Ambiental, 2001. Disponível em: <http:// www.semasa.sp.gov.br/Documentos/Publicar_Internet/trabaIhos/trabalho_72.pdf> Acesso em: 10 jun. 2019.

NEUMANN, B.; CALMON, A. P. S.; AGUIAR, M.M. Aplicação do ISA e Diagrama de Pareto como ferramentas de gestão do loteamento Lagoa Carapebus. Latin American Journal of Business Management, Taubaté, v. 4, n. 1, p.44-65, jun. 2013. Disponível em: <http://www.lajbm.net/index.php/journal/article/ view/120>. Acesso em: 19 fev. 2019.

PHILIPPI JR., A.; MARTINS, G. Águas de Abastecimento. In: PHILIPPI JR, A. (Ed). Saneamento, Saúde e Ambiente: Fundamentos para um desenvolvimento Sustentável. São Paulo: Manole, 2005. p. 117-180.

PINTO, L. P. et al. Condição ambiental do município de Diamante do Oeste - PR. Brazilian Journal Of Biosystems Engineering, Tupã, v. 10, n. 1, p.62-68, mar. 2016. Disponível em: <http:// seer.tupa.unesp.br/index.php/BIOENG/article/view/350/267>. Acesso em: 03 abr. 2019.
SÃO PAULO. Secretaria de Recursos Hídricos, Saneamento e Obras. ISA: Indicador de Salubridade Ambiental, manual básico. São Paulo, 1999. Edição SRHSO/Sabesp. São Paulo. 1999.

SILVA, N. V. S. As condições de Salubridade Ambiental das Comunidades Periurbanas da Bacia do Baixo Gramame: Diagnóstico e Proposição de Benefícios. 2006. 122 f. Dissertação (Mestrado em Engenharia Urbana), , Universidade Federal da Paraíba, Paraíba, 2006. Disponível: <http://www.hidro.ufcg.edu. br/twiki/pub/Rehisa/DocPublicacoes/Dissertao_Nayra_Setembro2006l.pdf> Acesso em: 16 mai. 2019.

WARTCHOW, D. Serviços de Abastecimento de água e de esgotamento sanitário. In: BRASIL, MINISTÉRIO DAS CIDADES. Lei Nacional de Saneamento Básico: Perspectiva para as políticas e a gestão dos serviços públicos - Livro II. Brasília: Ed, 2009. p. 273-283.

VALVASSORI, M. L.; ALEXANDRE, N. Z. Aplicação do Indicador de Salubridade Ambiental (ISA) para áreas urbanas. Revista Brasileira de Ciências Ambientais, Rio de Janeiro, v. 1, n. 25, p.1-19, set. 2012. Disponível em: <http://abes-dn.org.br/publicacoes/ rbciamb/PDFs/25-03_Materia_1_artigos321.pdf>. Acesso em: 03 abr. 2019. 